\title{
Antigen Binding
}

National Cancer Institute

\section{Source}

National Cancer Institute. Antigen Binding. NCI Thesaurus. Code C40474.

Antigen Binding Interaction involves specific and high affinity non-covalent interaction

(binding) of an endogenous antibody through intermolecular physical forces of attraction

and spatial complementarity with a soluble or particulate substance (antigen) that induces an immune response. 